\section{PC-037 STERILITY OF SINGLE USE VIALS WHEN ACCESSED USING A CLOSED SYSTEM TRANSFER DEVICE}

${ }^{1} \mathrm{JA}$ Schoenenberger Arnaiz, ${ }^{2} \mathrm{M}$ Nevot-Blanc* ${ }^{3} \mathrm{~A}$ Moroba-Estor, ${ }^{4} \mathrm{M}$ Gonzalez-Garcia, ${ }^{1} \mathrm{M}$ Martinez-Sogues, ${ }^{1} \mathrm{M}$ Gilabert-Sotoca. ${ }^{1}$ Hospital Universitari Arnau De Vilanova, Pharmacy, Lleida, Spain; ${ }^{2}$ Hospital Universitari Santa Maria, Pharmacy, Lleida, Spain; ${ }^{3}$ Irblleida, Clinical Trials, Lleida, Spain; ${ }^{4}$ Hospital Universitari Arnau De Vilanova, Microbiology, Lleida, Spain

\subsection{6/ejhpharm-2020-eahpconf.84}

Background and importance It is common practice for compounding facilities in Europe to retain partially used vials of physically stable drugs for future compounding needs; there are discrepancies in accepted beyond use dates (BUD). Canada NAPRA Model Standards specify a BUD of 6 hours based on the risk of microbial contamination for single use vials.

Aim and objectives To confirm that using a closed system transfer device (CSTD, ChemoLock), in a class II B2 BSC installed in a class $\mathrm{B}$ compliant environment, would prevent microbial contamination of simulated single use vials and maintain their sterility even after repeated access for up to 9 days.

Material and methods Under standard compounding procedures, 20 vials of growth media were accessed using ChemoLock vial adaptors. Aliquots $(1 \mathrm{~mL})$ were withdrawn and discarded from each vial at 0, 48, 96, 144, 168 and 216 hours. At the end of this 9 day period, samples were incubated in a microbiological laboratory. In order to test for growth of gram positive bacteria, gram negative bacteria, anaerobic microorganisms, yeast and mould, two types of growth media were used: 10 samples with tryptic soy broth (TSB) and 10 samples with fluid thioglycollate medium (FTM). Two temperature ranges $\left(20-25^{\circ} \mathrm{C}\right.$ and $\left.30-35^{\circ} \mathrm{C}\right)$ were used for incubation to ensure optimum growth conditions for different microorganisms. Negative and positive controls for each growth media were also used. Concurrent with testing, each positive control FTM vial was inoculated with one of the following American Type Culture Collection Stock (ATCC) strains: Clostridium sporogenes, Pseudomonas aeruginosa and Staphylococcus aureus, and incubated with the test samples. TSB vials were inoculated with one of the following ATCC stock strains: Bacillus subtilis, Aspergillus brasiliensis and Candida albicans.

Results No growth was observed in the negative controls or study samples after 7 days at $20-25^{\circ} \mathrm{C}$ or after 7 days at $30-$ $35^{\circ} \mathrm{C}$. All positive controls showed growth.

Conclusion and relevance The study results suggest that single use vial sterility is maintained for up to 9 days when accessed by a ChemoLock CSTD. Prolonged sterility may support BUD extension up 9 days. BUD extension may result in improved medication supply in times of shortage, increased efficiency and could prevent waste of high cost medicines.

\section{REFERENCES AND/OR ACKNOWLEDGEMENTS}

ICU Medical has sponsored this study

Conflict of interest Corporate sponsored research or other substantive relationships:

This research was sponsored by ICU Medical Inc, San Clemente, California, USA

\section{PC-038 PHYSICOCHEMICAL STABILITY OF THE BEVACIZUMAB BIOSIMILAR, ABP 215, IN INTRAVENOUS BAGS AFTER PREPARATION AND STORAGE}

J Seckute*, I Castellanos, S Bane. Amgen, Process Development, Cambridge, Massachusetts, USA

\subsection{6/ejhpharm-2020-eahpconf.85}

Background and importance ABP 215 (MVASI) is the first approved biosimilar to Avastin (bevacizumab reference product, RP). ABP 215 presents an alternative and effective treatment option. ABP 215 is administered intravenously after dilution in an infusion bag and confers its action by binding vascular endothelial growth factor (VEGF)-A and preventing its interaction with VEGF receptors on endothelial cells, resulting in angiogenesis inhibition. Extended physicochemical stability under in use conditions is valuable to enable administration flexibility by ensuring efficacy during handling conditions not covered by standard stability studies.

Aim and objectives The aim of the study was to evaluate the physicochemical stability of ABP 215 after dilution, storage and infusion during potentially worst case handling conditions.

Material and methods Low and high dose ABP 215 solutions were prepared in polyvinyl chloride and polyolefin intravenous (IV) bags. Prepared bags were stored at $2-8^{\circ} \mathrm{C}$ for 35 days and then stored at $30^{\circ} \mathrm{C}$ for 2 days, followed by IV infusion simulation. Stability indicating assays were selected to assess product quality on initial dilution into the IV bags, at prespecified time points during the $2-8^{\circ} \mathrm{C}$ refrigerated storage, $30^{\circ} \mathrm{C}$ storage, and prior and following the infusion. Stability indicating assays were selected to assess drug product quality. Size variants were assessed via size exclusion chromatography. Fragmentation was assessed via reduced capillary electrophoresis. Charge heterogeneity was assessed via cation exchange chromatography. Subvisible particle formation trends were assessed via subvisible particle testing and potential visible proteinaceous particle formation trends were assessed via visual inspection. Protein concentration was monitored for changes. Product quality consistency was evaluated via potency measurements.

Results After dilution, storage and infusion, ABP 215 solutions showed no changes outside of product acceptance criteria in molecular size or charge variants, fragmentation, particulate formation, protein concentration or potency.

Conclusion and relevance Across the evaluated worst case handling conditions, a robust set of stability indicating assays showed that ABP 215 product quality and activity remained within the product acceptance ranges over the examined extended storage.

\section{REFERENCES AND/OR ACKNOWLEDGEMENTS}

Conflict of interest Corporate sponsored research or other substantive relationships:

All authors are employees and stockholders of Amgen Inc. 